\title{
Adrenal pseudocyst mimicking a malignant tumor of the adrenal gland, about a case
}

\begin{abstract}
Background

Adrenal pseudocysts are exceptional, usually discovered fortuitously. The interest of this work resides in the particularity of this clinical case, because of its rarity, and the diagnostic difficulty that it posed. Moreover, the absence of specific clinical, biological and radiological signs of this adrenal pseudocyst entity which does not represent a usual tumor of the adrenal gland. So, through this case, the epidemiological, diagnostic and therapeutic aspects will be described.

Case presentation: Patient of 59 years old, with prior history of high blood pressure well controlled on monotherapy, reports since a year a pain in the right upper abdomen without any other associated signs. The physical examine is normal. Ultrasound and CT done revealed an adrenal mass refouling back closes organs, which was in favor of a malignant tumor of the adrenal gland. Biological examinations did not show any anomalies. The patient underwent an adrenalectomy without any complication. The histopathological analysis reveled the diagnosis of adrenalpseudocyst.

Conclusion: As showed without patient, clinical, biological and radiological signs of adrenalpseudocyst are not specific. Only histological examination can confirm the diagnosis and rule out malignancy.
\end{abstract}

Keywords: adrenal, histology, pseudocyst, surgery, tumor
Volume 6 Issue 3 - 2018

\author{
IRAQI Hinde, Labied Amal, SELLAY Sanae, \\ GHARBI Mohamed Elhassan \\ Department of Endocrinology Diabetology and Metabolic \\ diseases of Ibn Sina Hospital Rabat, Morocco
}

Correspondence: Labied Amal, Department of Endocrinology Diabetology and Metabolic diseases of Ibn Sina Hospital Rabat, groupe ksar souk, n I, kénitra, Morocco, Tel 00212655679522 , Emailamal_lab@hotmail.fr

Received: February 13, 2018 | Published: May 22, 2018
Abreviations: TDM, Tomodensitometrie; Mm, millimeter; $\mathrm{Cm}$, centimeter; $\mathrm{ACE}$, antigen carcinoembryonic; $\mathrm{F} / \mathrm{M}$, female/ Male

\section{Introduction}

Adrenal pseudocysts constitute an unusual adrenal tumor. Considering the increasful availability of the TDM, they represent $5.7 \%$ of adrenal incidentalomas. ${ }^{1}$

The cystic lesions of the adrenal gland are scarce with an estimated incidence of 0.064 to $0.18 \%{ }^{1-4}$ They can shift from malignant cystic tumors to pseudoscystics that represent 38 to $80 \%$ of all the adrenal cystic. ${ }^{3,5}$ Moreover, there are only 130 pseudocysts that have been reported in literature so far. ${ }^{1}$

We report a patient's case followed up for "adrenal incidentalomas", operated because of the tumor's size and the radiological suspicion of the malignancy. The pathologic study had concluded to an adrenal pseudocyst. Throughout this novel observation, we are going to review the epidemiological, clinical, pathological, and therapeutic of this little known condition.

\section{Case presentation}

Patient aged 59 years old, with prior history of high blood pressure well controlled on monotherapy, reports abdominal pains without any associated signs.

The abdominal ultrasound displays a right adrenal tumor mass: heterogeneous tissue with a very low vascularity at Doppler, measuring $70 \times 60 \mathrm{~mm}$, with pure limits, and regular contours. It also represents an intimate contact with the segment VI of the liver. Except the high blood pressure, no oriented symptoms towards an endocrine disorder have been reported.
A CT scan showed a right lesional adrenal process, well confined by lobulated contours, hypodense, containing greasy spots and calcifications (Figure 1), heterogeneously enhanced after a contrastive injection (Figure 2), measuring $71 \times 60 \times 55 \mathrm{~mm}$.

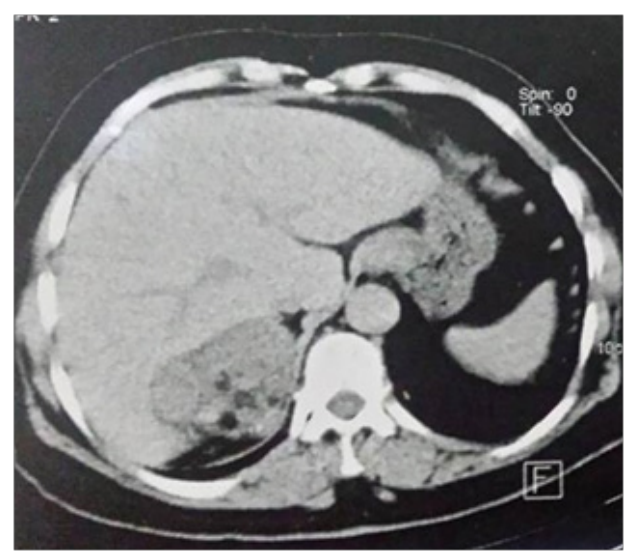

Figure I Abdominal CT scan showing the right adrenal mass hypodense containing greasy spots.

Frontwards, this process comes in contact with the inferior vena cava and segment VII of the liver without signs of invasion. Internally, he contacts the diaphragm's pillar with the loss of separation greasy edges in some place. The radiological aspect is highly suggestive of malignant adrenal tumor.

Biological tests are shown to be without anomalies, particularly in the context of exploring an adrenal incidentaloma. (Namely methoxylated derivatives and urinary free cortisol). 
Given the malignant radiological suspicion, and despite of getting a negative markers concerning the tumor (CA15-3, ACE, CA15-3, CA125, CA19-9 and alpha-fetoprotein), we conducted a research for primitive neoplasia by carrying pelvic ultrasound, mammography, bone scan and tiered CT. the whole was without the occurrence of any anomalies.

Given the size of the tumor and the radiological aspect of the malignant mass, a laparotomy was performed for right adrenalectomy, the mass removed weighs 73 grammes and measures $8 \times 6 \times 3 \mathrm{~cm}$ (Figure 3). The histopathologic study showed on the sections analyzed a whole enclosed section, an adrenal parenchyma with a cystic-like lesion bordered by a often thickened fibrous capsule. This lesion is made of vascular cavities often increased in size with the presence of hemorrhagic suffusions, fibrooedematous foci and amorphous fibrinous exudates. We also note the presence of deposits of hemosiderin and some macrophages, as well as foci of calcification and foci of adipose metaplasia. In conclusion, it is a morphological aspect of a pseudo adrenal cyst with no histological sign of malignancy.

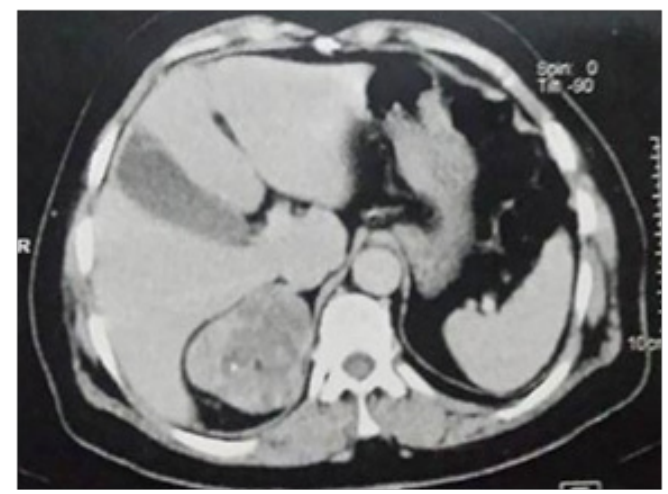

Figure 2 Scanner showing adrenal mass heterogeneously enhaced after contrast injection and containing calcifications.

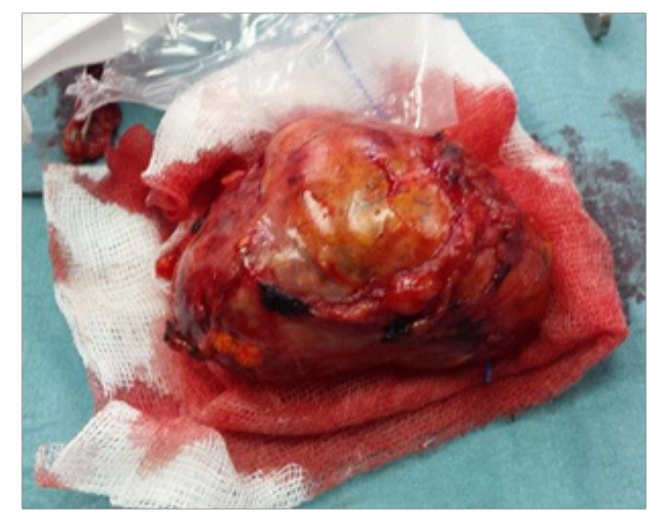

Figure 3 Macroscopic aspect of the surgical piece after complete resection.

The surgical follow-up was simple and the clinical monitoring at 3 months postoperative did not reveal any complications.

\section{Discussion}

Pseudocysts and adrenal cysts represent rare tumors of the adrenal gland usually discovered fortuitously at autopsy. They occur most often between the 4 th and 5 th decades. ${ }^{6}$ Our 59 -year-old patient is part of this age group, but cases have been reported at any age, ${ }^{7}$ and even during the neonatal period. ${ }^{1}$ We note a female predominance of this condition with a sex ratio of $3 \mathrm{~F} / 1 \mathrm{M}^{1,8}$

Histologically, adrenal cysts are classified in: ${ }^{1}$

I. Non-neoplastic cysts (classification of Barron and Emanuel):

a. Endothelial cyst (vascular): (incidence of $45 \%$ ), which includes:

b. Lymphangiomatous cyst (lymphangioma)

c. Angiomatous cyst

d. 2. Pseudocyst (39\%)

e. 3. Epithelial cyst $(9 \%)$

f. Parasitic cyst (7\%)

II. Neoplastic cyst (adrenal cystic carcinoma)

Pseudocysts are characterized by a fibrous wall without epithelial or endothelial structures and a reddish brown content of fibrin and necrotic debris. Pseudocysts with unusual variants have been reported, with intracystic fatty spots as in our case, or myelolipomatous metaplasia, both characteristic of a dermoid cyst; others have ectopic intra-thyroid tissue. ${ }^{1}$

Their true origin remains a mystery, and they can develop at the expense of the adrenal medulla or adrenal cortex, with two theories that may explain their pathogenesis: the first suggests that these lesions result from intra-adrenal hemorrhage caused by trauma, Sepsis or other form of shock. The initial lesion leads to the development of a cavity with cicatricial fibrous coating that grows over time., 2,4,8 Another theory suggests that these lesions are true cysts that have lost their cell lining due to inflammation and bleeding in the cyst. ${ }^{2,4,8}$

The pseudocysts are mostly unilateral and their size exceptionally exceeds $10 \mathrm{~cm}$. It is noted that $7 \%$ of all pseudocysts are malignant or potentially malignant and this risk increases with size, especially when it exceeds $6 \mathrm{~cm} \cdot{ }^{3,5}$

Most pseudocysts are asymptomatic, but in the case of a large pseudocyst the symptoms are due to the compression of the adjacent organs..$^{2,5}$

Thus, the three most frequent clinical manifestations are: abdominal pain, gastrointestinal symptoms (nausea and vomiting) and palpable mass. ${ }^{4}$ The acute abdomen table has been also described in some cases, due to intracystic hemorrhage, cyst rupture or infection. ${ }^{2,7}$

The authors have reported functional pseudocysts causing cushing syndrome $^{9}$ or pheochromocytoma ${ }^{7}$, or conversely pseudocysts responsible for adrenal insufficiency. ${ }^{10}$

In addition, the association between an adrenal cyst and several diseases is reported, including polycystic kidney disease, Beckwith Weidmann syndrome, Klippel Trenaunay syndrome ${ }^{1}$ and abdominal aortic aneurysm. ${ }^{1}$

The radiology confirms the cystic nature of the mass which appears at the scanner as a unilocular or multilobular cystic lesion. It can also occur as a mixed lesion or solid mass that may contain central calcifications that can mimic a true adrenal tumor. ${ }^{5}$ Like our case. Our patient's imagery showed a heterogeneous tissue adrenal mass with calcifications causing confusion initially with a malignant adrenal tumor. 
Treatment is determined by the size and symptoms related to the mass. Commonly the pseudocysts are presented to the surgeon if they are voluminous more than $5 \mathrm{~cm}$, in case of mass effect on the adjacents organs, if suspicious of malignant tumor and in case of hormonal secretion..$^{1,4,5}$

When surgery is proposed, laparoscopy is preferred to laparotomy regardless of the size of the tumor. ${ }^{3}$ Surgery may not be necessary if the lesions are labeled as small benign cystic lesions $(<4 \mathrm{~cm})$, and the patient can benefit from simple monitoring, which will be done by CT every 3 months for a period of 18 months. Beyond this, no radiological monitoring is discussed. ${ }^{1,3,5}$

Percutaneous puncture is reserved for small or recurrent cysts, as it exposes the risk of recurrence. ${ }^{1,2}$ In summary, Adrenal pseudocysts are a rare finding. Clinical and radiological characteristics of the tumor are not specific, and histopathological examination is essential in order to establish a definitive diagnosis. Surgery is necessary for symptomatic cases, large masses and in case of suspicion of malignancy.

The problem that remains to be asked in the absence of recommendations concerns the monitoring of these patients in postoperative care: with what? And at what pace?

\section{Acknowledgements and authors' contributions}

Pr HI: Conception and design, Drafting of the manuscript, Critical revision of the manuscript for important intellectual content

Dr AL: Conception and design, Drafting of the manuscript, Critical revision of the manuscript for important intellectual content, submission of the article

Dr SS: Critical revision of the manuscript for important intellectual content

Pr MG: Administrative technical or material support, Supervision

I would like to thank Dr Ahmed Anas Guerboub for his participation in the translation of this manuscript

\section{Conflict of interest}

The author declares no conflict of interest.

\section{Consent for publication}

Informed consent of the patient for publication in a medical journal was obtained in a document written in Arabic (mother tongue of the patient).

\section{References}

1. Nawin Kumar, Rajesh Parameshwaran Nair, Anuja Sinha. Giant adrenal pseudocyst presenting with hypertension: laparoscopic management. Med Res Chron. 2014;1(2):201-207.

2. Momiyama M, Matsuo K, Yoshida K, et al. A giant Adrenal Pseudocyst presenting with right hypochondralgia and fever: a case report. $\mathrm{J} \mathrm{Med}$ Case Rep. 2011;5:135.

3. Passoni S, Regusci L, Peloni G, et al. A Giant Adrenal Pseudocyst Mimicking an Adrenal Cancer: Case Report and Review of the Literature. Urol Int. 2013;91(2):245-248.

4. Singh A Kr, Dwevedi U, Mishra A Kr. Giant Adrenal Pseudocyst: A Rare Case Report. Sch J App Med Sci. 2013;1(4):342-343.

1. Ujam AB, Peters CJ, Tadrous PJ, et al. Adrenal pseudocyst: diagnosis and laparoscopie management, A case report. Int J Surg Case Rep. 2011;2(8):306-308.

5. Tazi N, Jahid A, Zouaidia F, et al. Pseudo kyste géant de la surrénale, à propos d'une nouvelle observation. African Journal of Urology. 2012;18(4):196-198.

6. Demir A, Tanidir Y, Kaya H, et al. A giant adrenal pseudocyst: Case report and review of the literature. Int Urol Nephrol. 2006;38(1):167-169.

7. Khilnani GC, Kumar A, Bammigatti C, et al. Hemorrhagic pseudocyst of the adrenal Gland causing acute abdominal pain. J Assoc Physicians India. 2008;56:379-380.

8. Brindley GV, Chisholm JT. Cystic tumors of the adrenal gland associated with Cushing's syndrome. Texas J Med. 1951;47:234-236.

9. Moore FP, Cemak EG. Adrenal cyst and adrenal insufficiency in an infant with fatal complication. J Pediatr. 1950;14:91-95. 